\title{
CONGRUENCE-INVARIANT MEASURES IN UNIFORM SPACES
}

\author{
BY \\ DAVID B. LEWIN
}

In the following paper, we shall define a congruence relation among subsets of a given locally compact uniform space $X$, and then demonstrate a method for constructing a well-behaved congruence-invariant measure on $X$. In particular: if $X$ is compact, then the measure of $X$ will be 1 . Another special case will be of interest: if every two points of $X$ have congruent neighborhoods, then the measure will be nontrivial and its support will be $X$. Thus, the method will yield Haar measure on a locally compact group.

In these respects, our construction is superior to that of Appert [1]: his measure must be zero on any countable space. Although his procedures can ke adjusted to obviate that defect, they do not seem to lead to general proofs of nontriviality in the cases mentioned above.

We shall employ the axiom of choice, and we shall not concern ourselves with uniqueness theorems. The methods of Banach [2] and Loomis [3] are more satisfactory in these respects. In compensation, however, we shall be able to demonstrate the existence and nontriviality of our measure for spaces which do not satisfy their conditions. Such is the case not only for most compact spaces, but also for a significant class of our "homogeneous" spaces. (This will be demonstrated in \$6.)

Our constructions generalize easily to any uniform space, but the results seem to have little content unless the space is at least locally totally-bounded. In the interests of stripping down the arguments, I shall restrict my attention somewhat further, and consider only locally compact spaces.

In the sequel, then, " $X$ " will denote a fixed locally compact uniform space. Its uniformities (entourages) will be denoted by " $u$ ", " $v$ ", etc. We shall use the following terminology:

$$
\begin{aligned}
& \text { If } Y \neq \varnothing \text { is a subset of } X, " d \operatorname{diam} Y \leqq u \text { " } \\
& \text { will mean } \forall_{y, y^{\prime}}^{\in Y},\left\langle y, y^{\prime}\right\rangle \in u \text {. } \\
& \text { If } Y \text { and } Z \text { are subsets, " } Y \text { and } Z \text { are separated" } \\
& \text { will mean } \exists u: \forall_{y}^{\in Y}, \forall_{z}^{\in Z},\langle y, z\rangle \notin u \text { and }\langle z, y\rangle \notin u \text {. }
\end{aligned}
$$

Received by the editors April 5, 1965 and, in revised form, March 1, 1966. 
An "outer measure" on $X$ will mean the usual: a function $\Gamma$ with domain the subsets of $X$ and range $\subseteq[0, \infty]$ such that $\Gamma(\varnothing)=0, \Gamma(Y) \leqq \Gamma(Z)$ when $Y \subseteq Z$, and $\Gamma\left(\bigcup_{1}^{\infty} Y_{i}\right) \leqq \Sigma_{1}^{\infty} \Gamma\left(Y_{i}\right)$.

$$
\begin{aligned}
& \text { An outer measure } \Gamma \text { is "Carathéodory" when } \\
& Y \text { and } Z \text { separated } \rightarrow \Gamma(Y \cup Z)=\Gamma(Y)+\Gamma(Z) \text {. }
\end{aligned}
$$

A well-known theorem states that if $X$ is a metric space and $\Gamma$ is Carathéodory, then every open set is measurable. In analogous fashion, one proves for any uniform space $X$ :

$\Gamma$ Carathéodory: then every uniformly continuous real-valued function on $X$ is measurable.

Hence, for our locally compact $X$,

$\Gamma$ Carathéodory; then every continuous real-valued function with compact support is measurable.

For every such function must be uniformly continuous (the proof, by contradiction, is straightforward).

We now define a congruence relation among the subsets of $X$. We fix a uniform basis $\mathfrak{U}$.

" $Y$ is weakly congruent to $Z[Y \sim Z]$ " will mean:

$\exists$ a 1-1 map $f$ of $Y$ onto $Z$ such that

$\forall \underset{u}{\in U} \forall_{y, y^{\prime}}^{\in Y},\left\langle y, y^{\prime}\right\rangle \in u \leftrightarrow\left\langle f(y), f\left(y^{\prime}\right)\right\rangle \in u$.

" $Y$ is congruent to $Z[Y \approx Z]$ " will mean:

$\exists$ a uniformity $v$ such that $v(Y) \sim v(Z)$ via some map $f$ such that $f(Y)=Z$.

One notes that if $Y$ is open and congruent to $Z$, then $Z$ is also open.

We now proceed to the construction of a congruence-invariant Carathéodory outer measure on $X$. Some of the following choices and restrictions are arbitrary and unnecessary so far as obtaining such a measure is concerned; they seem natural, however.

We fix an open subset $X_{0} \neq \varnothing$ of $X$ with compact closure.

Definition 2.1. If $Y \neq \varnothing$ is a subset of $X$, and $u \in \mathfrak{U}$, set

$$
\begin{aligned}
N(u, Y)= & \min \left\{n: \exists P_{1}, \cdots, P_{n}, \text { open, with } Y \subseteq \bigcup_{1}^{n} P_{i} \text { and } \operatorname{diam} P_{i} \leqq u\right\} \\
& \text { if any such finite covering of } Y \text { exists. } \\
N(u, Y)= & \infty \text { otherwise. }
\end{aligned}
$$

Set $N(u, \varnothing)=0$.

This covering function has the following obvious properties: 

a. $Y \subseteq Z \rightarrow N(u, Y) \leqq N(u, Z)$,
b. $N(u, Y \cup Z) \leqq N(u, Y)+N(u, Z)$.
c. if $Y$ and $Z$ are separated by $v_{0}$, then $\forall u \subseteq v_{0}, N(u, Y \cup Z)=N(u, Y)+N(u, Z)$.

Since $X_{0} \neq \varnothing$ has compact closure, $0<N\left(u, X_{0}\right)<\infty$.

Definition 2.2. Set $t(u, Y)=N(u, Y) / N\left(u, X_{0}\right)$.

The function $t(u, Y)$ evidently has the same properties as does $N(u, Y)$. In addition, we note that, if $Z \subseteq X_{0}$, then $t(u, Z) \leqq 1$.

Now, for each $Z \subseteq X_{0}$, let $I_{Z}$ be a replica of the unit interval [0,1]. Let $S=\prod_{z \subseteq X_{0}} I_{Z}$ be the (compact) Tychonoff product space.

For each $u \in \mathfrak{U}$, we regard $t_{u}=\langle t(u, Z)\rangle$ as a point of $S .\left\{t_{u}\right\}$ is then a directed system of points in a compact space.

CHOICES 2.31. Choose any accumulation point $t_{0}$ of this system.

2.32. Choose any subsystem $\left\{t_{u_{\alpha}}\right\}$ such that

$$
\forall Z \subseteq X_{0}, t\left(u_{\alpha}, Z\right) \underset{\alpha}{\rightarrow} t_{0}(Z)
$$

Construction 2.4. Extend the domain of the set-function $t_{0}$ over all subsets of $X$ by defining

$$
\begin{aligned}
t_{0}(Y) & =\lim _{\alpha} \inf t\left(u_{\alpha}, Y\right) \\
& =\lim _{\alpha} \underset{\beta>\alpha}{\operatorname{glb}}\left\{t\left(u_{\beta}, Y\right)\right\} .
\end{aligned}
$$

The set function $t_{0}$, thus extended, has the following properties:

a. $Y \subseteq Z \rightarrow t_{0}(Y) \leqq t_{0}(Z)$,

b. $\quad Y$ and $Z$ separated $\rightarrow t_{0}(Y \cup Z) \geqq t_{0}(Y)+t_{0}(Z)$,

c. $Z_{1}, \cdots, Z_{n} \subseteq X_{0}$; then $t_{0}\left(\bigcup_{1}^{n} Z_{i}\right) \leqq \Sigma_{1}^{n} t_{0}\left(Z_{i}\right)$,

d. $t_{0}(\varnothing)=0 ; t_{0}\left(X_{0}\right)=1$.

(Property c, above, is not generally valid for denumerably infinite sums and unions within $X_{0}$.)

We are now ready for our outer measure:

DEFINITION 2.5. Set $\Gamma(Y)=\operatorname{glb}\left\{\Sigma_{1}^{\infty} t_{0}\left(0_{i}\right): 0_{i}\right.$ open, $\left.Y \subseteq \bigcup_{1}^{\infty} 0_{i}\right\}$.

THEOREM 2.51. $\Gamma$ is an outer measure.

Standard Proof. Note that $t_{0}(\varnothing)=0$ implies $\Gamma(\varnothing)=0$.

THEOREM 2.52. $\Gamma$ is Carathéodory.

Proof. We are to show: $Y$ and $Z$ separated $\rightarrow \Gamma(Y \cup Z) \geqq \Gamma(Y)+\Gamma(Z)$. Let $u_{0}$ separate $Y$ and $Z$. Find $v_{0}$ symmetrical with $v_{0}^{3} \subseteq u_{0}$. Let $A=\bigcup_{Y}$ interior $v_{0}(y), B=\bigcup_{z}$ interior $v_{0}(z)$. Then $A$ and $B$ are evidently separated by $v_{0}$.

Now, given $\varepsilon>0$, find $\left\{0_{i}\right\}$ with $Y \cup Z \subseteq \bigcup 0_{i}$ and $\Gamma(Y \cup Z)+\varepsilon \geqq \sum t_{0}\left(0_{i}\right)$. 
Let $A_{i}=A \cap 0_{i}, B_{i}=B \cap 0_{i}$. Then (2.41a) $t_{0}\left(0_{i}\right) \geqq t_{0}\left(A_{i} \cup B_{i}\right)$. But, since $A_{i}$ and $B_{i}$ are separated, $t_{0}\left(A_{i} \cup B_{i}\right) \geqq t_{0}\left(A_{i}\right)+t_{0}\left(B_{i}\right)(2.41 \mathrm{~b})$. Finally, $A_{i}$ and $B_{i}$ are open, $Y \subseteq \bigcup A_{i}$, and $Z \subseteq \bigcup B_{i}$. Then, by construction, $\Sigma t_{0}\left(A_{i}\right) \geqq \Gamma(Y)$ and $\Sigma t_{0}\left(B_{i}\right) \geqq \Gamma(Z)$. Since we may assume $\Gamma(Y \cup Z)<\infty$ (otherwise the proof is trivial), we can put these facts together and get $\Gamma(Y \cup Z)+\varepsilon \geqq \Gamma(Y)+\Gamma(Z)$. But $\varepsilon$ was arbitrary. q.e.d.

LEMMA 2.531. If $Y \approx Y^{\prime}$, then $t_{0}(Y)=t_{0}\left(Y^{\prime}\right)$.

Proof. Find $v_{0}$ and $f$ as required by 1.32. Choose $\alpha_{0}$ such that $u_{\alpha_{0}}^{2} \subseteq v_{0}$. Consider any $\alpha>\alpha_{0}$. Suppose $N\left(u_{\alpha}, Y\right)=n<\infty$. Let $Y \subseteq \bigcup_{1}^{n} P_{i}, P_{i}$ open, $\operatorname{diam} P_{i} \leqq u_{\alpha}$. By the minimality of $n$, each $P_{i}$ must intersect $Y$; and since $\operatorname{diam} P_{i} \leqq u_{\alpha}, P_{i} \subseteq u_{\alpha}(Y) . A$ fortiori, $P_{i} \subseteq v_{0}(Y)$ and hence $\operatorname{diam} f\left(P_{i}\right) \leqq u_{\alpha}$ (1.31).

Further, $u_{\alpha}\left(P_{i}\right) \subseteq u_{\alpha}^{2}(Y) \subseteq v_{0}(Y)$. Hence $f$ maps $u_{\alpha}\left(P_{i}\right)$ 1-1 onto $u_{\alpha}\left(f\left(P_{i}\right)\right)$, and $P_{i} \approx f\left(P_{i}\right)$ [cf. (1.32)]. Then $f\left(P_{i}\right)$ must be open. Finally, $Y^{\prime} \subseteq \bigcup_{1}^{n} f\left(P_{i}\right)$. But then $N\left(u_{\alpha}, Y^{\prime}\right)$ is not infinite, and in fact is $\leqq n=N\left(u_{\alpha}, Y\right)$.

Reversing the roles of $Y$ and $Y^{\prime}$, via $f^{-1}$, we infer: $\forall \alpha>\alpha_{0}, N\left(u_{\alpha}, Y\right)=N\left(u_{\alpha}, Y^{\prime}\right)$, whether or not this quantity is finite. The lemma follows, by construction of $t_{0}$.

THEOREM 2.532. If $Y \approx Y^{\prime}, \Gamma(Y)=\Gamma\left(Y^{\prime}\right)$.

Proof. Let $v_{0}, f, \alpha_{0}$ be as in 2.531. Let $A=\bigcup_{y \in Y}$ interior $u_{\alpha_{0}}(y), A^{\prime}=\bigcup_{y^{\prime} \in Y^{\prime}}$ interior $u_{\alpha_{0}}\left(y^{\prime}\right)$. Then it is easily seen that $A^{\prime}=f(A)$ and $A \approx A^{\prime}$ via $f$, since $u_{\alpha_{0}}(A) \subseteq v_{0}(Y)$.

Now, given $\varepsilon>0$, find $\left\{0_{i}\right\}$ with $Y \subseteq \bigcup_{1}^{\infty} 0_{i}$ and $\Gamma(Y)+\varepsilon \geqq \Sigma t_{0}\left(0_{i}\right)$. Let $A_{i}=A \cap 0_{i}$. Then $t_{0}\left(0_{i}\right) \geqq t_{0}\left(A_{i}\right)$. Let $A_{i}^{\prime}=f\left(A_{i}\right)$. Then $A_{i}^{\prime} \approx A_{i}$ and $t_{0}\left(A_{i}\right)=t_{0}\left(A_{i}^{\prime}\right)$ via the preceding lemma. $A_{i}$ being open and $\approx A_{i}^{\prime}, A_{i}^{\prime}$ is also open and $Y^{\prime} \subseteq \bigcup A_{i}^{\prime}$. Hence $\Sigma t_{0}\left(A_{i}^{\prime}\right) \geqq \Gamma\left(Y^{\prime}\right)$. Putting this together and recalling that $\varepsilon$ was arbitrary, we infer $\Gamma(Y) \geqq \Gamma\left(Y^{\prime}\right)$. Reversing the roles of $Y$ and $Y^{\prime}$, we obtain the reverse inequality, q.e.d.

Our $\Gamma$ is thus a congruence-invariant Carathéodory outer measure. We come now to a crucial theorem, which justifies the construction of $\left\{u_{\alpha}\right\}$ [rather than taking $t_{0}=\lim \inf _{u \in \mathfrak{M}} t(u$,$) everywhere].$

THEOREM 3.1. If $Y \subseteq X_{0}$ and $Y$ is compact, then

$$
\Gamma(Y)=\operatorname{glb}\left\{t_{0}(A): A \text { open, } Y \subseteq A\right\} .
$$

Proof. It will be recalled that we assumed $X_{0}$ to be open. Let $\gamma=$ the glb of the theorem. $\Gamma(Y) \leqq \gamma$ by construction of $\Gamma$.

Now, given $\varepsilon>0$, find $0_{i}$ with $Y \subseteq \bigcup_{1}^{\infty} 0_{i}$ and $\Gamma(Y)+\varepsilon \geqq \Sigma_{1}^{\infty} t_{0}\left(0_{i}\right)$. Let $A_{i}=X_{0} \cap 0_{i}$. Then $t_{0}\left(0_{i}\right) \geqq t_{0}\left(A_{i}\right)$ and $\Gamma(Y)+\varepsilon \geqq \Sigma_{1}^{\infty} t_{0}\left(A_{i}\right)$. Y being compact and $A_{i}$ open, let $Y \subseteq \bigcup_{1}^{n} A_{i}$. Let $A=\bigcup_{1}^{n} A_{i}$. Then $\Gamma(Y)+\varepsilon \geqq \Sigma_{1}^{n} t_{0}\left(A_{i}\right) \geqq t_{0}(A)$ $[$ N.B. $(2.41 \mathrm{c})] \geqq \gamma$. But $\varepsilon$ was arbitrary. q.e.d. 
COROllary 3.11. $Y$ compact $\subseteq X_{0}$; then $\Gamma(Y) \geqq t_{0}(Y)$.

THEOREM 3.2. If $X$ is compact and $X_{0}$ is taken $=X$, then

$$
\Gamma(X)=1 \text {. }
$$

This is an immediate consequence of 2.5 and 3.11, since $t_{0}(X)=1$.

We list further theorems, the proofs of which are straightforward.

(3.31) $\forall Y, \Gamma(Y)=\operatorname{glb}\{\Gamma(A): A$ open, $Y \subseteq A\}$.

(3.311) If $X$ is a metric space, $\Gamma$ is regular. Further: $\forall Y, \exists a G_{\delta}$-set $G$ such that $Y \subseteq G, \Gamma(Y)=\Gamma(G)$.

(3.32) If $t_{0}(Y)<\infty$, then $Y$ is totally bounded.

(3.321) If $\Gamma(Y)<\infty$, then $\forall u, \exists y_{i} \in Y: Y \subseteq \bigcup_{1}^{\infty} u\left(y_{i}\right)$.

We turn now to the consideration of what we shall call " $\mathfrak{U}$-homogeneous", spaces.

DEFINITION 4.1. A uniform space $X$ is $\mathfrak{H}$-homogeneous (given the uniform basis $\mathfrak{U}$ ) when $\forall_{x}^{\in X}, \underset{y}{X},\{x\} \approx\{y\}$ [see (1.32)].

As before, we shall continue to restrict our attention to locally compact spaces, assuming $X_{0}$ chosen, $t_{0},\left\{u_{\alpha}\right\}$ and $\Gamma$ constructed.

Lemma 4.21. If $X$ is $\mathfrak{U}$-homogeneous, then $t_{0}(0)>0$ for all open $0 \subseteq X, 0 \neq \varnothing$.

Proof. Suppose not: $y_{0} \in 0_{0}$ and $t_{0}\left(0_{0}\right)=0$. Then, by the assumption of homogeneity, we can find, for each $y \in X$, an open set $0_{y}$ with $y \in 0_{y}, t_{0}\left(0_{y}\right)=0$ [see 2.531]. Now $\bar{X}_{0}$ is compact. Find $y_{1}, \cdots, y_{n}$ with $\bar{X}_{0} \subseteq \bigcup_{1}^{n} 0_{y_{i}}$. Let $Y_{i}=X_{0} \cap 0_{y_{i}}$. Then $X_{0}=\bigcup_{1}^{n} Y_{i}$. Hence $[2.41 \mathrm{c}] 1=t_{0}\left(X_{0}\right) \leqq \Sigma_{1}^{n} t_{0}\left(Y_{i}\right) \leqq \Sigma t_{0}\left(0_{y_{i}}\right)$, a contradiction. q.e.d.

THEOREM 4.22. If $X$ is $\mathfrak{U}$-homogeneous, then $\Gamma$ is nontrivial and its support is $X$ :

$$
\begin{aligned}
& \forall x, \forall u, \exists \text { a measurable (compact) neighborhood } \\
& C \text { of } x \text { with } C \subseteq u(x), 0<\Gamma(C) \leqq 1
\end{aligned}
$$

Proof. Fix $x_{0} \in X_{0}$. Given any $x$ and any $u$. Find $v_{0}, f$, with $v_{0}\left(x_{0}\right) \sim v_{0}(x)$ via $f, f\left(x_{0}\right)=x$. Choose $v_{1} \in \mathfrak{U}$ with $v_{1}^{2} \leqq v_{0} \cap u$ and $v_{1}\left(x_{0}\right) \subseteq X_{0}$.

Let $\phi$ be a real-valued uniformly continuous function with $\phi\left(x_{0}\right)=1, \phi \equiv 0$ off interior $v_{1}\left(x_{0}\right)$. Let $C_{0}=\{y: \phi(y) \geqq 1 / 2\}$. $C_{0}$ is a compact neighborhood of $x_{0}$.

Since $C_{0} \subseteq X_{0}, \Gamma\left(C_{0}\right) \leqq t_{0}\left(X_{0}\right)=1$. Since $C_{0}$ is a neighborhood of $x_{0}, t_{0}\left(C_{0}\right)>0$ [4.21]. And since $C_{0}$ is compact and $\subseteq X_{0}, \Gamma\left(C_{0}\right) \geqq t_{0}\left(C_{0}\right)$ (3.11). Hence $0<\Gamma\left(C_{0}\right) \leqq 1$.

Let $C=f\left(C_{0}\right) . C$ is then the required neighborhood of $x$. For $C_{0} \subseteq v_{1}\left(x_{0}\right)$, whence $v_{1}\left(C_{0}\right) \subseteq v_{1}^{2}\left(x_{0}\right) \subseteq v_{0}\left(x_{0}\right) \cdots$ hence $v_{1}\left(C_{0}\right) \sim v_{1}(C)$ (recalling that $v_{1}$ was chosen $\in \mathfrak{U}$ ), and $C_{0} \approx C$. 
From the latter, we infer that $\Gamma(C)=\Gamma\left(C_{0}\right)$, so that $0<\Gamma(C) \leqq 1$; also that $C$ is a compact neighborhood of $x$. We also have $C \subseteq v_{1}(x) \subseteq u(x)$, as required.

Finally, the function $\phi^{\prime}$ on $X$, defined by $\phi^{\prime}(y)=\phi f^{-1}(y)\left[y \in v_{0}(x)\right], \phi^{\prime}(y)=0$ otherwise, is uniformly continuous; hence $C=\left\{y: \phi^{\prime}(y) \geqq 1 / 2\right\}$ is measurable. q.e.d.

Thus, in particular, $\Gamma$ is outer Haar measure on any locally compact group [see (1.23)].

5. Some remarks on generalizing the preceding work. Let $X$ be any uniform space, $X_{0} \neq \varnothing$ a totally bounded subset, $\mathfrak{U}$ a given basis, and $\boldsymbol{P}=\{P\}$ any family of subsets of $X$ such that (1) for any $u$, a finite number of $P$ of diam $\leqq u$ cover any totally bounded $Y$ and (2) $P \in \boldsymbol{P}, P \approx Q \rightarrow Q \in \boldsymbol{P}$. (For instance, we could take $\boldsymbol{P}=\{$ closed sets $\},\{$ all subsets $\},\{\mathfrak{U}$-spheres $\}$, et al.)

Then the work of (2) above carries through, and we can obtain a Carathéodory congruence-invariant outer measure on $X$, with all the properties of (3.31. etc.) 3.1 is amended to require $Y$ compact and $\subseteq$ interior $X_{0}$.

6. We now examine our idea of $\mathfrak{U}$-homogeneity in the light of the work of Banach [2] and Loomis [3].

Banach derives a nontrivial measure from an abstract congruence relation on a locally compact [metric] space. In order to show that his measure is congruenceinvariant, he makes (among others) two assumptions, which can be considerably weakened as follows: $\exists$ a base $\Omega$ of open sets with compact closures, such that $Z \in \Omega, Z^{\prime} \cong Z \rightarrow Z^{\prime} \in \Omega$, satisfying also (B1) if $Z \in \Omega$, then $\left\{Z^{\prime}: Z^{\prime} \cong Z\right\}$ covers $X$ and (B2) if $Y \subseteq X, Y^{\prime} \cong Y$, then $\exists \varepsilon>0$ : whenever $Z_{n} \in \Omega$, diam $Z_{n} \leqq \varepsilon, Y \subseteq \bigcup Z_{n}$, we can find $Z_{n}^{\prime} \cong Z_{n}$ with $Y^{\prime} \subseteq \bigcup Z_{n}^{\prime}$. We shall call such a base a "Banach base". One notes that if $X$ is any [uniform] space whose topology is discrete, $X$ is $\mathfrak{U}$ homogeneous for any $\mathfrak{U}$, but also $X$ has the Banach base $\Omega=\{\{x\}: x \in X\}$ with respect to $\mathfrak{U}$-congruence.

Loomis considers uniform spaces $X$ possessing a special sort of uniform basis $\mathfrak{B}$. The uniformities of $\mathfrak{B}$ are symmetrical; $\forall u \in \mathfrak{B}, x \in X, u(x)$ is totally bounded and (L1) the minimum number of $u$-spheres needed to cover a $v$-sphere is independent of the center of the $v$-sphere. He imposes one other restriction which we shall ignore, calling any such $\mathfrak{B}$ a "Loomis basis".

We note now that any open subset of a locally compact group is a locally compact $\mathfrak{U}$-homogeneous space, as a subspace of the group, for any basis $\mathfrak{U}$. One also observes that if $X$ is a metric space and $\mathfrak{U}$ is any basis, weak congruence is equivalent to isometry and the congruence and weak congruence relations are independent of any particular $\mathfrak{U}$ selected.

For each $n=1, \cdots$, let $I_{n}$ be the open subset $\left(n, n+2^{-n}\right)$ of the real line. Let $X=\bigcup I_{n}$; consider $X$ as a metric subspace of the real line. Obviously $X$ cannot possess a Banach base. A bit of straightforward calculation also shows that $X$ cannot possess a Loomis basis. It is clear that by constructing $X$ with more care, 
we could make it totally bounded and, if we go into two dimensions, the connected interior of a region in the plane with rectifiable boundary (construct, over a rectangular base, a picket fence whose pickets dwindle in thickness and height).

We can also construct spaces which are not obvious open subspaces of topological groups. For example, take $X$ a well-disjointed union of randomly skew dwindling open arcs of constant and equal curvature embedded in Euclidean $n$-space, with the induced metric. So, as asserted earlier, a significant class of locally compact $\mathfrak{U}$-homogeneous spaces is not amenable to the techniques of Banach or Loomis. An essential feature of these spaces is that they are not uniformly $G$-homogeneous; clearly any uniformly $\mathfrak{U}$-homogeneous space has a Banach base and a Loomis basis. One conjectures that a compact $\mathfrak{U}$-homogeneous space should be uniformly $\mathfrak{U}$-homogeneous, although it does not seem so evident that this could be proved without using the axiom of choice.

\section{REFERENCES}

1. A. Appert, Mesures normales dans les espaces distanciés, Bull. Sci. Math. (2) 60 (1936), 329-352; 368-380.

2. S. Banach, On Haar's measure, Note I in S. Saks, Theory of the integral, pp. 314-319, Monogr. Mat., Warsaw, 1937.

3. L. H. Loomis, Haar measure in uniform structures, Duke Math. J. 16 (1949), 193-208.

University of California, Music Department, Berkeley, California 